\title{
On some hereditable shape properties
}

\author{
by KARoL BorsuK (Tarszawa)
}

\begin{abstract}
Let $A$ bo a compactum. A compactum $X$ lying in a space $M \in A R(m)$ is said to be $A$-movable if for every neighborhood $U$ of $X$ in $M$ there exists a neighborhood $U_{0}$ of $X$ (in $M$ ) such that for every neighborhood $V$ of $X$ (in $M$ ) each map $a: A \rightarrow U_{0}$ is homotopic in $U$ to a map with all values in $V$. It is shown that the choice of the space $M \in \mathrm{AR}(\mathfrak{M})$ containing $X$ is immaterial and that the $A$-movability is a hereditable shape property of $X$. Some relations between the $A$-movability and the formerly known concepts of the movability and of the $n$-movability are established.
\end{abstract}

Among shape properties (concerning the notions belonging to the shape theory see, for instance [2]) a special role is played by the property of movability (see [1], p. 137), because several important theorems (as theorems corresponding in the shape theory to theorems of Hurewicz [4] and of Whitehead [5]) are true for movable compacta, but they fail if one ounits the hypothesis of movability.

The aim of this note is to introduce a family of properties analogous to the movability, and to prove that they are hereditable shape properties.

Let $A$ and $X$ be two compacta. We may assume that $X$ is a subset of a space $M \in \mathrm{AR}(\mathfrak{M})$. Let us say that $X$ is $A$-movable in $M$ if for every neighborhood $U$ of $X$ in $M$ there exists a neighborhood $U_{0}$ of $X$ (in $M$ ) such that for every neighborhood $O$ of $X$ (in $M$ ) and for every map $\alpha: A \rightarrow U_{0}$ there is a homotopy

such that

$$
\varphi: A \times\langle 0,1\rangle \rightarrow U
$$

$$
\varphi(p, 0)=\alpha(p) \text { and } \varphi(p, 1) \in \theta \text { for every point } p \in A .
$$

ExAMPLES. (a) If $A$ is a contractible in itself compactum, then every compactum $X \subset M \in \mathrm{AR}(\mathfrak{M})$ is $A$-movable in $M$. In fact, for every neighborhood $U$ of $X$ in $M$ there exists a neighborhood $U_{0} \subset U$ of $X$ in $M$ such that for every point $x \in U_{0}$ there is a point $\lambda(x) \in X$ such that $x$ and $\lambda(x)$ can be joined by an arc lying in $U$. Since $A$ is contractible in itself, each map $\alpha: A \rightarrow U_{0}$ is homotopic in $U$ to a map $\alpha^{\prime}$ mapping $A$ onto a single point $x \in U_{0}$. Then $\alpha^{\prime}$ is homotopic in $U$ to the map $a^{\prime \prime}$ mapping $A$ onto the point $\lambda(x) \in X$, hence $\alpha$ is homotopic in $U$ to the map $\alpha^{\prime \prime}$ with values lying in each neighborhood $\theta$ of $X$. 
(b) If $X$ is a dyadic solenoid of Van Dantzig, lying in the euclidean 3 -space $M=E^{3}$, and if $A$ is the circle $S^{1}$, then $X$ is not $A$-movable in $M$. In fact, there exists in $E^{3}$ a sequence of solid tori $T_{1}, T_{2}, \ldots$ such that $T_{k+1}$ lies in the interior of $T_{k}$ and its oriented core is homologous in $T_{k}$ to the twice described core of $T_{k}$ and that $X=\bigcap_{k=1}^{\infty} T_{k}$. One sees easily that any homeomorphism a mapping $A$ onto the core of $T_{k}$ with $k>1$ is not homotopic in $T_{1}$ to any map with values in $T_{k+1}$.

Now we prove the following

(2) THEOREM. Let $A, X, Y$ be compacta and $M, N$ be $\operatorname{AR}(\mathfrak{M})$-spaces such that $X \subset M, Y \subset N$. If $X$ is A-movable in $M$ and if $\operatorname{Sh}(X) \geqslant \operatorname{Sh}(Y)$, then $Y$ is $A$-movable in $N$.

Proof. The hypothesis $\operatorname{Sh}(X) \geqslant \operatorname{Sh}(Y)$ means that there exist two fundamental sequences:

such that

$$
\underline{f}=\left\{f_{k}, X, Y\right\}_{M, N} \quad \text { and } \quad \underline{g}=\left\{g_{k}, Y, X\right\}_{N, M}
$$

$$
\underline{f g} \simeq \underline{i}_{Y, N}
$$

Consider a neighborhood $V$ of $Y$ in $N$. Then there exists a neighborhood $U$ of $X$ in $M$ and an index $k_{1}$ such that

$$
f_{k} / U \simeq f_{k_{1}} / U \text { in } V \text { for every } k \geqslant k_{1} .
$$

Moreover, (3) implies that there exists a neighborhood $V_{1} \subset V$ of $Y$ in $N$ and an index $k_{2} \geqslant k_{1}$ such that

$$
f_{k} g_{k} / V_{1} \simeq i / V_{1} \quad \text { in } V \quad \text { for every } k \geqslant k_{2} .
$$

Since $X$ is $A$-movable in $M$, there exists a neighborhood $U_{0}$ of $X$ in $M$ such that for every neighborhood $\hat{U}$ of $X$ in $M$ and for every map a: $A \rightarrow U_{0}$ there is a homotopy

$$
\varphi: A \times\langle 0,1\rangle \rightarrow U
$$

satisfying condition (1). Since $g$ is a fundamental sequence, there exists a neighborhood $V_{0} \subset V_{1}$ of $Y$ in $N$ and an index $k_{3} \geqslant k_{2}$ such that

$$
g_{k}\left(V_{0}\right) \subset U_{0} \quad \text { for every } k \geqslant k_{3} \text {. }
$$

Now let $\beta$ be a map of $A$ into $V_{0}$ and let $V$ be an arbitrary neighborhood of $Y$ in $N$. Since $f$ is a fundamental sequence, there is a neighborhood $U$ of $X$ in $M I$ and an index $k_{1} \geqslant k_{3}$ such that

Setting

$$
f_{k}(\theta) \subset \hat{V} \quad \text { for every } k \geqslant k_{4} \text {. }
$$

$$
a(p)=g_{k_{4}} \beta(p) \quad \text { for every point } p \in A
$$


one gets (in view of (5) and the inequality $k_{4} \geqslant k_{3}$ ) a map $\alpha: A \rightarrow U_{0}$. Consequently there is a homotopy $\varphi: A \times\langle 0,1\rangle \rightarrow U$ satisfying condition (1). It follows by (1), (3) and (4) that the formula

$$
\psi^{\prime}(p, t)=f_{k_{4}} \varphi(p, t) \quad \text { for every }(p, t) \epsilon A \times\langle\mathbf{0}, \mathbf{1}\rangle,
$$

defines a homotopy

$$
\psi^{\prime}: A \times\langle\mathbf{0}, \mathbf{1}\rangle \rightarrow V .
$$

Using (1), (9), (8) and (7), we infer that

(10) $\psi^{\prime}(p, 0)=f_{k_{4}} g_{k_{4}} \beta(p)$ and $\quad \psi^{\prime}(p, 1) \epsilon \hat{V} \quad$ for every point $p \in A$.

Since $\beta(A) \subset V_{0} \subset V_{1}$, we infer by (5) and (6) that $f_{k_{4}} g_{k_{4}} \beta \simeq \beta$ in $V$, i.e. there exists a homotopy

such that

$$
\psi^{\prime \prime}: A \times\langle\mathbf{0}, \mathbf{1}\rangle \rightarrow V
$$

$$
\psi^{\prime \prime}(p, 0)=\beta(p) \quad \text { and } \quad \psi^{\prime \prime}(p, 1)=f_{k_{4}} g_{k_{4}} \beta(p)
$$

for every point $p \in A$.

Setting

$$
\begin{array}{ll}
\psi(p, t)=\psi^{\prime \prime}(p, 2 t) & \text { for }(p, t) \in A \times\left\langle 0, \frac{1}{2}\right\rangle, \\
\psi(p, t)=\psi^{\prime}(p, 2 t-1) & \text { for }(p, t) \in A \times\left\langle\frac{1}{2}, 1\right\rangle,
\end{array}
$$

we get a homotopy

$$
\psi: A \times\langle 0,1\rangle \rightarrow V .
$$

Using (10) and (11) we infer that

$\psi(p, 0)=\beta(p) \quad$ and $\quad \psi(p, 1)=\psi^{\prime}(p, 1) \epsilon \hat{V} \quad$ for every point $p \in A$.

Thus $Y$ is $A$-movable in $N$ and the proof of theorem (2) is finished.

(12) CoRoLi.ARY. The A-movability of $X$ in $M$ does not depend on the choice of the space $M \in \operatorname{AR}(\mathfrak{M})$ containing $X$.

It follows that the words "in $M$ " are for the property of $A$-movability superfluous. Thus, instead to say " $X$ is $A$-movable in $M$ " we can say shortly that $\boldsymbol{X}$ is $\boldsymbol{A}$-movable.

(13) Corollary. A-movability is a hereditable shape-property, that is if $X$ is A-movable and $\operatorname{Sh}(X) \geqslant \operatorname{Sh}(Y)$, then $Y$ is A-movable.

In [3], p. 859 a property called the n-movability has been introduced for every $n=1,2, \ldots$ Let us recall its definition:

A compactum $X$ is said to be $n$-movable if it is homeomorphic to a subset $X^{\prime}$ of the Hilbert cube $Q$ such that for every neighborhood $U$ of $X^{\prime}$ in $Q$ there exists a neighborhood $U_{0}$ of $X^{\prime}$ in $Q$ such that each compactum 
$B \subset U_{0}$ with $\operatorname{dim} B \leqslant n$ is homotopic in $U$ to a subset of an arbitrarily given neighborhood $U$ of $X^{\prime}$ in $Q$. One knows that:

(14) n-movability is a hereditable shape-property.

(15) Every movable compactum is n-movable for $n=1,2, \ldots$

(16) Solenoids of Van Dantzig are not 1-movable.

Now we prove the following

(17) THEOREM. If $X$ is an n-movable compactum, then $X$ is $A$-movable for every compactum $A$ with $\operatorname{dim} A \leqslant n$.

Proof. Using theorem (2), we may assume that $X \subset Q$. Let $U$ be a noighborhood of $X$ in $Q$. Since $X$ is $n$-movable, there exists an open neighborhood $U_{0}$ of $X$ (in $Q$ ) such that every at most $n$-dimensional compactum lying in $U_{0}$ is homotopic in $U$ to a subset of an arbitrarily given neighborhood $U$ of $X$ (in $Q$ ).

Consider a map $a: A \rightarrow U_{0}$. It is well known that there exists a homeomorphism $\alpha^{\prime}: A \rightarrow U_{0}$ homotopic to $\alpha$ in $U$. Then $\operatorname{dim} \alpha^{\prime}(A) \leqslant n$ and consequently there is a homotopy carrying the set $\alpha^{\prime}(A)$ in $U$ onto a subset of $\hat{U}$. Hence $a$ is homotopic in $U$ to map with all values in $\hat{U}$ and the proof of theorem (17) is finished.

(18) CoRollary. If $X$ is movable, then $X$ is movable for every compactum $\boldsymbol{A}$.

(19) Problem. Let $S^{k}$ denote the $k$-dimensional sphere. Does there exist a compactum $X$ which is $S^{k}$-movable for $k=1,2, \ldots, n$, but is not n-movable?

\section{References}

[1] K. Borsuk, On movable compacta, Fund. Math. 66 (1969), p. 137-146.

[2] - On the concept of shape for metrizable spaces, Bull. Acad. Polon. Sci. 18 (1970), p. 127-132.

[3] - On the n-movability, ibidem 20 (1972), p. 859-865.

[4] K. Kuperberg, An isomorphism theorem of Hurewicz type in the Borsuk's theory of shape, Fund. Math. 77 (1973), p. 21-32.

[5] M. Moszynska, The Whitehead theorem in the theory of shape, ibidem (to appear). 